\title{
Regime alimentar para ganho compensatório de ovinos em confinamento: desempenho produtivo e morfometria do rúmen e do intestino delgado
}

\author{
[Diet for compensatory gain of feedlot lambs: performance and morphometry \\ of the rumen and small intestine] \\ G.H. Nóbrega ${ }^{1}$, M.F. Cézar ${ }^{2}$, O.B. Sousa², J.M. Pereira Filho², W.H. Sousa ${ }^{3}$, M.G.G. Cunha ${ }^{3}$, \\ M.A. Cordão ${ }^{1}$, R.C. Ferreira ${ }^{1}$, J.R.S. Santos ${ }^{1}$ \\ ${ }^{1}$ Aluno de pós-graduação - Universidade Federal de Campina Grande - UFCG - Patos, PB \\ ${ }^{2}$ Universidade Federal de Campina Grande - Patos, PB \\ ${ }^{3}$ EMEPA-PB, Soledade, PB
}

\begin{abstract}
RESUMO
Objetivou-se avaliar o efeito do regime alimentar para ganho compensatório sobre o desempenho produtivo, o peso do corpo vazio, o peso dos componentes abióticos, o peso e a proporção do TGI e a morfometria do rúmen e do intestino delgado de cordeiros terminados em confinamento. Para tanto, foram utilizados 40 cordeiros Santa Inês, machos não castrados, alojados em baias individuais. O confinamento foi dividido em dois períodos distintos de 42 dias: um de restrição alimentar, com quatro tratamentos $(0,20,40$ e $60 \%$ de restrição), e outro de realimentação, em que todos os animais foram realimentados sem restrição. Ao final do período de confinamento, os cordeiros foram abatidos para mensuração do peso do TGI, do peso dos componentes abióticos e para obtenção dos fragmentos do rúmen e do intestino delgado, os quais foram posteriormente utilizados na confecção das lâminas histológicas usadas para avaliação da morfometria das papilas ruminais e das vilosidades e criptas do intestino $(\mu \mathrm{m})$. As variáveis relativas ao desempenho, além de peso ao abate, peso do corpo vazio e peso do TGI, diminuíram à medida que se aumentou o nível de restrição prévia. A largura das papilas ruminais diminuiu, variando de 555,7 a 470,3 $\mu \mathrm{m}$; a área das vilosidades do intestino aumentou, variando de 81042,8 a $92033,7 \mu \mathrm{m}^{2}$. O regime alimentar para ganho compensatório diminuiu o ganho de peso, o peso ao abate, o peso do corpo vazio e o peso do trato gastrintestinal de cordeiros Santa Inês, terminados em confinamento, além de afetar a morfometria das papilas ruminais e das vilosidades do intestino delgado.
\end{abstract}

Palavras-chave: ovino, papilas ruminais, restrição alimentar, vilosidades intestinais

\begin{abstract}
The aim was evaluate the effect of diet for compensatory growth on performance, empty weight body, weight of abiotic components, weight and proportion of TGI and morphology of the rumen and small intestine of lambs feedlot. Thus, we used 40 Santa Inês non castrated male lambs, housed in individual pens. The containment was divided into two distinct periods of 42 days: a food restriction, with four treatments (0,20, 40 and 60\% restriction), and a feedback loop, where all the animals were fed again without restriction. At the end of the period of confinement they were slaughtered to measure the weight of TGI, weight and abiotic components for obtaining fragments of the rumen and small intestine, which were subsequently used for the preparation of histological slides used to evaluate the morphology of the papillae rumen and the intestinal villi and crypts $(\mu \mathrm{m})$. The variables for performance, slaughter weight, empty body weight and weight of TGI decreased as they increased the level of prior restraint. The width of the rumen papillae decreased ranging from 555.7 to $470.3 \mu \mathrm{m}$; the area of increased intestinal villi ranged from 81042.8 to $92033.7 \mathrm{\mu m}^{2}$. The diet for compensatory gain decreases weight gain, slaughter weight, empty body weight and the weight of the gastrointestinal tract of Santa Ines lambs, besides affecting the morphology of rumen papillae and villi of the small intestine.
\end{abstract}

Keywords: ovine, food restriction, intestinal villi, rumen papillae

Recebido em 27 de abril de 2013

Aceito em 19 de fevereiro de 2014

E-mail: ghnobrega@hotmail.com 


\section{INTRODUÇÃO}

Atualmente, a produção de ovinos no Brasil está em ritmo crescente e vem se caracterizando como uma atividade de grande importância cultural, social e econômica para a região Nordeste, em cujo desenvolvimento desempenha papel crucial. A população nacional de ovinos é de 17,7 milhões de cabeças, com destaque para a região Nordeste, que possui o maior rebanho do país, com o efetivo de 10,1 milhões de cabeças, o que corresponde a $57,2 \%$ do total nacional (IBGE, 2011).

No entanto, nessa região, a época seca se traduz num grande entrave na produção animal, com a escassez de alimento e/ou a diminuição no valor nutritivo das forragens, o que dificulta o ciclo normal da demanda desses animais e, consequentemente, leva à necessidade da implantação de confinamento nessa época, a fim de incrementar os custos da produção. Nesse sentido, o regime alimentar para ganho compensatório seria uma alternativa, uma vez que o crescimento dos animais se torna mais acelerado quando o alimento volta a ser abundante, após um período de restrição alimentar, tornando-se uma interessante ferramenta para a obtenção de boas respostas na produtividade desses animais. Esse regime alimentar está relacionado ao maior consumo de alimentos, à melhor conversão alimentar e também ao melhor aproveitamento dos nutrientes, resultante da modificação dos órgãos internos (Ryan, 1990).

Sistemas de alimentação devem ser estudados, pois é sabido que mudanças na alimentação, durante o período de crescimento do animal, alteram a ingestão e a digestibilidade e, dessa forma, podem influenciar no desenvolvimento dos órgãos (Jenkins, 1993). Para obter o máximo de suas funções, os órgãos e as vísceras modificam sua estrutura de acordo com o tipo de alimentação que o animal está ingerindo. Segundo Kamalzadeh et al. (1998), órgãos e vísceras, em comparação a outras partes do corpo do animal, apresentam diferentes velocidades de crescimento e são influenciados principalmente pela composição química da dieta e pelo seu nível energético.

O epitélio gastrintestinal é responsável por muitas funções fisiológicas, incluindo a digestão, a absorção, o transporte e o metabolismo dos nutrientes; a digestão e absorção estão relacionadas com o desenvolvimento da papila e da vilosidade (Xu et al., 2009). As características do rúmen podem ser influenciadas quanto ao alimento fornecido, pois, de acordo com Martens et al. (2012), ovinos inicialmente alimentados com uma dieta com baixa energia e, posteriormente, com concentrado sofrem alterações no epitélio ruminal, particularmente no tamanho das papilas, em adaptação a mudanças nos parâmetros ruminais, como pH, concentração de ácidos graxos e pressão osmótica. O epitélio do intestino delgado também pode mudar sua estrutura de acordo com o alimento ingerido, já que ele parece se adaptar para satisfazer as necessidades nutricionais do animal (Zitnan et al., 2008).

O objetivo da realização deste trabalho foi avaliar a influência do regime alimentar para ganho compensatório (restrição alimentar, seguida por realimentação) sobre o peso animal, o ganho de peso, o consumo (matéria seca, proteína e energia), o peso do corpo vazio, o peso dos componentes abióticos, o peso e a proporção do trato gastrintestinal, bem como na morfometria das papilas ruminais, das vilosidades e das criptas do intestino delgado de cordeiros Santa Inês, terminados em confinamento.

\section{MATERIAL E MÉTODOS}

Para realização do experimento, foram utilizados 40 ovinos Santa Inês, machos inteiros, desmamados, com média \pm desvio-padrão de $17 \pm 1,7 \mathrm{~kg}$ de peso corporal e 100 dias de idade. No início do experimento, os animais foram identificados, tratados contra ecto e endoparasitas e vacinados contra clostridioses. Em seguida, foram alojados em baias individuais, com dimensões de 1,0 x $1,2 \mathrm{~m}$, alocadas em galpão com piso de cimento coberto com telhas de barro, equipadas com comedouros e bebedouros.

Os animais eram alimentados duas vezes ao dia, às sete e às 15 horas. A dieta experimental (Tab. 1), na forma de ração completa, foi formulada com base nas exigências desses animais para um ganho de $250 \mathrm{~g}$ por dia, segundo as recomendações do NRC (National..., 1985). 
Tabela 1. Proporção dos ingredientes e composição bromatológica da ração experimental

\begin{tabular}{|c|c|}
\hline Ingredientes & Proporção (\%) \\
\hline Feno de Tifton & 30,0 \\
\hline Milho moído & 47,0 \\
\hline Farelo de soja & 16,5 \\
\hline Farelo de trigo & 4,0 \\
\hline Calcário & 1,5 \\
\hline Sal mineral & 1,0 \\
\hline \multicolumn{2}{|l|}{$\begin{array}{l}\text { Composição } \\
\text { bromatológica }\end{array}$} \\
\hline Matéria seca & 90,07 \\
\hline Proteína bruta ${ }^{1}$ & 16,25 \\
\hline Extrato etéreo $^{1}$ & 3,17 \\
\hline $\begin{array}{l}\text { Energia metabolizável } \\
(\text { Mcal } / \mathrm{kg} \text { de } \mathrm{MS})^{2}\end{array}$ & 2,82 \\
\hline $\begin{array}{l}\text { Fibra em detergente } \\
\text { neutro }\end{array}$ & 63,84 \\
\hline Matéria mineral $^{1}$ & 6,14 \\
\hline \multicolumn{2}{|c|}{$\begin{array}{l}{ }^{1} \% \text { em relação à matéria seca; }{ }^{2} \mathrm{EM}=\mathrm{ED} \text { x } 0,82 \text {, em } \\
\text { que EM é a energia metabolizável, ED é a energia } \\
\text { digestível da dieta }(3,44 \mathrm{Mcal} / \mathrm{kg} \text { de MS, Borburema, } \\
2010) \text { e } 0,82 \text { é a metabolizabilidade da dieta } \\
\text { (National..., 2007). }\end{array}$} \\
\hline
\end{tabular}

O período experimental foi de 98 dias, incluindo 14 dias iniciais de adaptação dos animais às instalações, ao manejo e à dieta, sendo o restante dividido em dois períodos distintos de 42 dias cada: um de restrição alimentar (do primeiro ao $42^{\circ}$ dia) e outro de realimentação $\left(43^{\circ}\right.$ ao $84^{\circ}$ dia). No período de restrição alimentar, os animais foram divididos em quatro tratamentos: $0,20,40$ e $60 \%$ de restrição. Os animais do tratamento $0 \%$ recebiam alimentação à vontade, com reajuste diário que permitia sobra de $10 \%$, o que garantia o consumo voluntário; já aqueles dos demais tratamentos seguiram um regime de restrição alimentar, de 20, 40 e $60 \%$, em relação ao tratamento sem restrição $(0 \%)$. No período de realimentação, todos os animais, de todos os tratamentos, foram realimentados sem restrição, ou seja, à vontade.

Os animais eram pesados a cada 14 dias, para avaliação do ganho de peso, sempre precedida de jejum sólido de 16 horas. A avaliação do consumo era feita diariamente, mediante pesagem do alimento oferecido e das sobras. Amostras compostas da dieta e das sobras por animal eram elaboradas para posteriores análises de matéria seca (MS), matéria orgânica (MO), proteína bruta (PB) e energia bruta (EB), segundo as metodologias descritas por Silva e Queiroz (2002).

Ao final do período de confinamento (restrição seguida de realimentação), os cordeiros foram submetidos a jejum de 16 horas de líquidos e de 24 horas de sólidos; em seguida, foram pesados para obtenção do peso vivo ao abate (PA). Os animais foram abatidos e, logo após eviscerados. Posteriormente, foram pesados $\mathrm{o}$ trato gastrintestinal (TGI), a bexiga e a vesícula biliar, os quais foram esvaziados, limpos e novamente pesados, para obtenção do peso do TGI, do peso dos componentes abióticos (PCA) (conteúdo do TGI, da bexiga e da vesícula biliar) e do peso do corpo vazio $(\mathrm{PCV}=\mathrm{PA}$ - PCA).

A obtenção dos fragmentos do rúmen e do intestino delgado foi realizada imediatamente após o abate e o esvaziamento das vísceras. Com o objetivo de se evitarem alterações pós-morte no material, as amostras foram rapidamente imersas em formol tamponado e fixadas por 24 horas. Após a fixação, as peças foram lavadas em água corrente e mantidas em álcool $70 \%$ até o momento do processamento histológico para inclusão em parafina. Fragmentos teciduais de $3 \mathrm{~mm}$ de espessura foram desidratados em concentrações crescentes de álcool (70 a 100\%), diafanizados em xilol e, logo após, incluídos em parafina a $58-60^{\circ} \mathrm{C}$. Os blocos de parafina foram levados ao micrótomo rotativo para a obtenção de cortes histológicos com $5 \mu \mathrm{m}$ de espessura. Em seguida, foram corados pela técnica de hematoxilina-eosina (HE), e as lâminas montadas com lamínula sobre entellan, conforme Samuelson (2007).

Nas secções de rúmen, foram mensuradas altura, largura e área das papilas; e no intestino delgado, foram medidas altura, largura, superfície, área das vilosidades e profundidade das criptas (todos em $\mu \mathrm{m})$. A captação de imagens e as análises foram realizadas com auxílio dos softwares computacionais Q-Capture e ImagePro Express 6.0, acoplados a um microscópio de bancada, Olympus BX41, com objetiva de $4 \mathrm{x}$ e de 10x, para os cortes de rúmen e intestino, respectivamente. A relação altura das vilosidades:profundidade das criptas foi determinada pela razão entre a altura das vilosidades e a profundidade das criptas do intestino. 
O delineamento experimental utilizado foi $o$ inteiramente ao acaso, com quatro tratamentos (considerando as dietas do período de restrição $0,20,40$ e $60 \%$ ) e 10 repetições. As análises estatísticas foram realizadas por meio de análise de variância, de regressão e de correlação, segundo os procedimentos PROC GLM, PROC REG e PROC CORR do SAS (Statistical..., 2003).

\section{RESULTADOS E DISCUSSÃO}

A variável peso inicial não revelou diferenças entre os vários tratamentos, o que significa que os animais foram agrupados obedecendo ao princípio básico de uniformidade das amostras, conforme demonstra a Tab. 2.

Houve diminuição das variáveis ganho de peso total e ganho de peso médio diário, quando se aumentou o nível de restrição prévia. O período de realimentação não foi suficiente para compensar o menor ganho de peso dos animais, obtido no período de restrição prévia, ou seja, não houve ganho compensatório para essas variáveis. Esse mesmo comportamento foi observado por Almeida et al. (2011), ao trabalharem com ovinos Santa Inês, mais maduros, em confinamento. Os autores concluíram que a proximidade do peso à maturidade diminui a compensação do peso. A resposta ao regime de ganho compensatório depende de vários fatores, como idade do animal, tipo, severidade e duração da restrição e do nível de ingestão (Ben Salem e Smith, 2008). Assim, o nível de consumo dos animais deste trabalho, também decrescente em função do aumento do nível de restrição prévia, determinou a diminuição do ganho de peso.

Tabela 2. Médias e desvios-padrão do peso inicial (PI), do ganho de peso médio diário e total (GPMD e GPT), do consumo de matéria seca, da proteína bruta e da energia bruta (CMS, CPB e CEB) de cordeiros Santa Inês, submetidos ao regime alimentar para ganho compensatório, terminados em confinamento

\begin{tabular}{|c|c|c|c|c|c|}
\hline \multirow{2}{*}{ Variáveis } & \multicolumn{4}{|c|}{ Tratamentos } & \multirow[b]{2}{*}{$\mathrm{p}$} \\
\hline & $0 \%$ & $20 \%$ & $40 \%$ & $60 \%$ & \\
\hline PI (kg) & $20,3 \pm 2,8$ & $20,9 \pm 1,7$ & $20,3 \pm 1,8$ & $19,1 \pm 1,5$ & ns \\
\hline GPT (kg) & $21,0 \pm 3,1$ & $18,7 \pm 4,0$ & $17,7 \pm 3,0$ & $14,5 \pm 2,3$ & $<0,01^{1}$ \\
\hline GPMD $(\mathrm{g})$ & $250,2 \pm 36,7$ & $223,1 \pm 47,5$ & $210,4 \pm 35,3$ & $172,1 \pm 27,7$ & $<0,01^{2}$ \\
\hline CMS (kg) & $1,355 \pm 0,20$ & $1,211 \pm 0,11$ & $1,067 \pm 0,09$ & $0,873 \pm 0,07$ & $<0,01^{3}$ \\
\hline CPB $(\mathrm{kg})$ & $0,223 \pm 0,03$ & $0,198 \pm 0,02$ & $0,175 \pm 0,02$ & $0,143 \pm 0,01$ & $<0,01^{4}$ \\
\hline CEB (Mcal/kg) & $6,396 \pm 0,93$ & $5,716 \pm 0,51$ & $5,036 \pm 0,42$ & $4,121 \pm 0,35$ & $<0,01^{5}$ \\
\hline
\end{tabular}

${ }^{1} \mathrm{y}=21,08530-0,10379 \mathrm{x} \quad\left(\mathrm{r}^{2}=0,37\right) ;{ }^{2} \mathrm{y}=251,01800-1,23560 \mathrm{x} \quad\left(\mathrm{r}^{2}=0,37\right) ; \quad{ }^{3} \mathrm{y}=1,36496-0,00795 \mathrm{x} \quad\left(\mathrm{r}^{2}=0,68\right) ; \quad{ }^{4} \mathrm{y}=0,22421-0,00132 \mathrm{x}$ $\left(\mathrm{r}^{2}=0,67\right) ;{ }^{5} \mathrm{y}=6.44339-0.03753 \mathrm{x}\left(\mathrm{r}^{2}=0,68\right)$.

Os consumos (MS, PB e EB) diminuíram linearmente à medida que se aumentou o nível de restrição prévia. Esse resultado foi semelhante ao observado por Homem Júnior et al. (2010), em cordeiros Santa Inês em confinamento. Os autores ressaltaram que o consumo é o maior determinante do desempenho animal. Dessa forma, caso houvesse aumento do consumo durante a realimentação, haveria ganhos equivalentes ou até mesmo superiores aos dos animais do regime alimentar à vontade.

Ao final do experimento, os animais dos tratamentos com restrição prévia, ou seja, 20, 40 e $60 \%$, deixaram de ganhar, respectivamente, 2,$4 ; 4,6$ e $8,2 \mathrm{~kg}$. A economia de alimento, no entanto, foi de 10,$6 ; 21,3$ e $35,6 \%$ em relação àqueles sem restrição prévia, o que, de acordo com Homem Júnior et al. (2007), indica que restrições mais severas não são recomendadas, pois podem prejudicar o desempenho de cordeiros. Entretanto, níveis menores podem ser adotados como prática de manejo nutricional, para períodos de escassez de alimento ou para ovinos que serão submetidos a confinamento, como forma de reduzir o tempo de confinamento, o consumo total de alimento e, consequentemente, os custos com alimentação.

As variáveis peso dos componentes abióticos e proporção do TGI relativo ao PCV (Tab. 3) não sofreram influência do período de restrição prévia $(\mathrm{P}>0,05)$. $\mathrm{O}$ conteúdo do trato gastrintestinal, que constitui a maior parte dos componentes abióticos (em torno de 98\%), é influenciado pela relação volumoso:concentrado e pelo tipo de volumoso (Moreno et al., 2011), que, quando mais fibroso, passa mais tempo no 
TGI, durante o período de jejum (Medeiros et al., 2008), e, assim, pode alterar o peso desses componentes. Entretanto, o volumoso e sua relação com concentrado mantiveram-se constantes nos tratamentos e ao longo de todo o período experimental, ou seja, na fase de restrição prévia e de realimentação, o que explica a não alteração do peso dos componentes abióticos em função do regime alimentar.

Tabela 3. Médias e desvios-padrão das variáveis peso ao abate (PA), peso do corpo vazio (PCV), peso dos componentes abióticos (PCA), peso do trato gastrintestinal (TGI) e a proporção do TGI relativo ao PCV de cordeiros Santa Inês, submetidos ao regime alimentar para ganho compensatório, terminados em confinamento

\begin{tabular}{|c|c|c|c|c|c|}
\hline \multirow{2}{*}{ Variáveis } & \multicolumn{4}{|c|}{ Tratamentos } & \multirow[b]{2}{*}{$\mathrm{p}$} \\
\hline & $0 \%$ & $20 \%$ & $40 \%$ & $60 \%$ & \\
\hline PA (kg) & $42,7 \pm 5,1$ & $40,6 \pm 4,2$ & $38,7 \pm 3,1$ & $34,7 \pm 2,9$ & $<0,01^{1}$ \\
\hline PCV (kg) & $37,7 \pm 4,4$ & $35,2 \pm 3,4$ & $34,0 \pm 2,7$ & $29,9 \pm 2,3$ & $<0,01^{2}$ \\
\hline PCA (kg) & $5,0 \pm 1,0$ & $5,4 \pm 1,2$ & $4,8 \pm 1,1$ & $4,8 \pm 1,0$ & ns \\
\hline TGI (kg) & $3,5 \pm 0,6$ & $3,2 \pm 0,3$ & $3,0 \pm 0,3$ & $2,7 \pm 0,4$ & $<0,01^{3}$ \\
\hline TGI (\%) & $9,2 \pm 0,8$ & $8,9 \pm 0,7$ & $9,1 \pm 1,3$ & $9,0 \pm 0,9$ & ns \\
\hline
\end{tabular}

Owens et al. (1993) afirmam que diferentes tecidos se desenvolvem em ondas específicas de crescimento, ou seja, alguns crescem e amadurecem antes dos outros, e, nesse sentido, alegam que o peso do TGI pode mudar dentro de algumas semanas - uma vez que a atividade metabólica desses tecidos é considerável (Zitnan et al., 2008) -, mesmo após reduzir seu tamanho, para economizar energia, em decorrência de várias semanas de baixa ingestão, fato que explica a manutenção da proporção do TGI em relação ao PCV.

O PA diminuiu com o aumento da restrição prévia $(\mathrm{P}<0,05)$, como consequência direta da redução observada no consumo e no ganho de peso. O PCV sofreu influência do regime alimentar, com efeito linear negativo $(\mathrm{P}<0,05)$ em relação ao aumento da restrição prévia. Resultado semelhante foi observado por Medeiros et al. (2008), em pesquisa com ovinos Morada Nova, em confinamento, na qual concluíram que o aumento dos níveis de concentrado na dieta contribui para elevar o PCV dos cordeiros. O peso do TGI também diminuiu com o aumento da restrição prévia $(\mathrm{P}<0,05)$, seguindo o mesmo comportamento do PCV, o que está de acordo com Yamamoto et al. (2004), quando dizem que o peso dos não componentes da carcaça acompanha o aumento do peso do animal, e com a afirmação de Moreno et al. (2011) de que mudanças na alimentação, durante o período de crescimento do animal, alteram a ingestão e, consequentemente, influencia o desenvolvimento dos órgãos.

As variáveis, altura e área das papilas ruminais (Fig. 1) não foram influenciadas pelo regime alimentar ( $\mathrm{P}>0,05)$, no entanto a largura das papilas diminuiu, de forma linear $(\mathrm{P}<0,01)$, à medida que se aumentou o nível de restrição prévia, ou seja, a menor quantidade de alimento que os animais receberam no período de restrição inibiu parcialmente o desenvolvimento das papilas ruminais (Tab. 4). O tamanho das papilas diminui durante um período de subalimentação e aumenta quando a oferta alimentar se normaliza, porém esse aumento ocorre de forma lenta, chegando a um tamanho máximo em torno de 50 a 60 dias (Martens et al., 2012).

Os valores obtidos para as variáveis altura e largura das papilas ruminais foram, respectivamente, menores e maiores que os obtidos por Norouzian et al. (2011), com médias de $1407,6 \mu \mathrm{m}$ e $260,6 \mu \mathrm{m}$, quando trabalharam com cordeiros recém-desmamados e testaram diferentes dietas para inclusão de sólidos na alimentação. São resultados diversos, que podem variar em função da idade ao desmame, da quantidade, tipo e teor de energia do alimento (Sun et al., 2011) e da estimulação física por ele provocado (Álvarez-Rodríguez et al., 2012). 
Tabela 4. Médias e desvios-padrão da altura (APR), largura (LPR) e área das papilas (ARPR) do rúmen, da altura (AVI), largura (LVI), superfície (SVI) e área das vilosidades (ARVI), da profundidade das criptas (PCI) e da relação altura das vilosidades:profundidade das criptas (AVI:PCI) do intestino de cordeiros Santa Inês, submetidos ao regime alimentar para ganho compensatório, terminados em confinamento

\begin{tabular}{|c|c|c|c|c|c|}
\hline \multirow{2}{*}{ Variáveis } & \multicolumn{4}{|c|}{ Tratamentos } & \multirow[b]{2}{*}{$\mathrm{p}$} \\
\hline & $0 \%$ & $20 \%$ & $40 \%$ & $60 \%$ & \\
\hline $\mathrm{APR}(\mu \mathrm{m})$ & $968,0 \pm 146,9$ & $983,3 \pm 82,0$ & $944,0 \pm 126,6$ & $969,6 \pm 122,1$ & ns \\
\hline $\mathrm{LPR}(\mu \mathrm{m})$ & $555,7 \pm 54,2$ & $498,4 \pm 36,9$ & $508,7 \pm 53,1$ & $470,3 \pm 32,7$ & $<0,01^{1}$ \\
\hline $\begin{array}{l}\text { ARPR } \\
\left(\mu \mathrm{m}^{2}\right)\end{array}$ & $508049,1 \pm 125517,7$ & $468096,9 \pm 42006,7$ & $448676,5 \pm 74110,9$ & $442050,7 \pm 61303,5$ & ns \\
\hline AVI $(\mu \mathrm{m})$ & $442,7 \pm 85,5$ & $458,9 \pm 50,0$ & $494,6 \pm 71,8$ & $478,1 \pm 47,0$ & ns \\
\hline LVI $(\mu \mathrm{m})$ & $196,1 \pm 25,0$ & $186,1 \pm 12,0$ & $189,2 \pm 24,0$ & $201,1 \pm 15,0$ & ns \\
\hline $\operatorname{SVI}(\mu \mathrm{m})$ & $1006,3 \pm 164,3$ & $1030,9 \pm 90,5$ & $1107,4 \pm 143,7$ & $1080,0 \pm 95,1$ & ns \\
\hline $\begin{array}{l}\text { ARVI } \\
\left(\mu \mathrm{m}^{2}\right)\end{array}$ & $81042,8 \pm 15150,9$ & $80580,7 \pm 7864,7$ & $88096,4 \pm 16468,5$ & $92033,7 \pm 14442,1$ & $<0,05^{2}$ \\
\hline PCI $(\mu \mathrm{m})$ & $358,4 \pm 83,3$ & $399,0 \pm 57,9$ & $401,9 \pm 84,8$ & $405,2 \pm 70,4$ & ns \\
\hline AVI:PCI & $1,3 \pm 0,3$ & $1,2 \pm 0,2$ & $1,3 \pm 0,2$ & $1,2 \pm 0,3$ & ns \\
\hline
\end{tabular}

$\mathrm{y}=545,19376-1,23062 \mathrm{x}\left(\mathrm{r}^{2}=0,27\right) ;{ }^{2} \mathrm{y}=79482+200,98185 \times\left(\mathrm{r}^{2}=0,10\right)$.

Observa-se também na Tab. 4 que as variáveis altura, largura, área das vilosidades, profundidade das criptas intestinais (Fig. 2) e a relação altura das vilosidades:profundidade das criptas não sofreram influência do regime alimentar $(\mathrm{P}>0,05)$. Em trabalho com caprinos, alimentados com diferentes níveis de amido, Wang et al. (2009) também não observaram

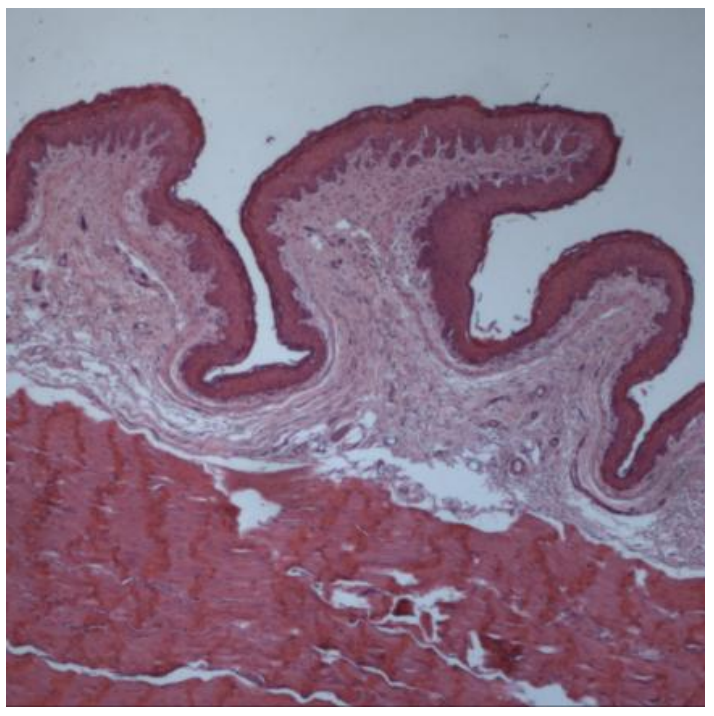

Figura 1. Papilas ruminais $(4 \mathrm{x} ; \mathrm{HE})$ de cordeiros Santa Inês, submetidos ao regime alimentar para ganho compensatório, terminados em confinamento.

No entanto, a área das vilosidades do intestino aumentou, de forma linear $(\mathrm{P}<0,05)$, quando se aumentou o nível de restrição prévia, mudanças na estrutura da superfície mucosa do intestino delgado e afirmaram que alterações na estrutura das vilosidades determinam a capacidade digestiva e absortiva do intestino delgado, ou seja, vilosidades mais longas e maior relação altura de vilosidade:profundidade da cripta resultam em maior capacidade de absorção de nutrientes pelo intestino.

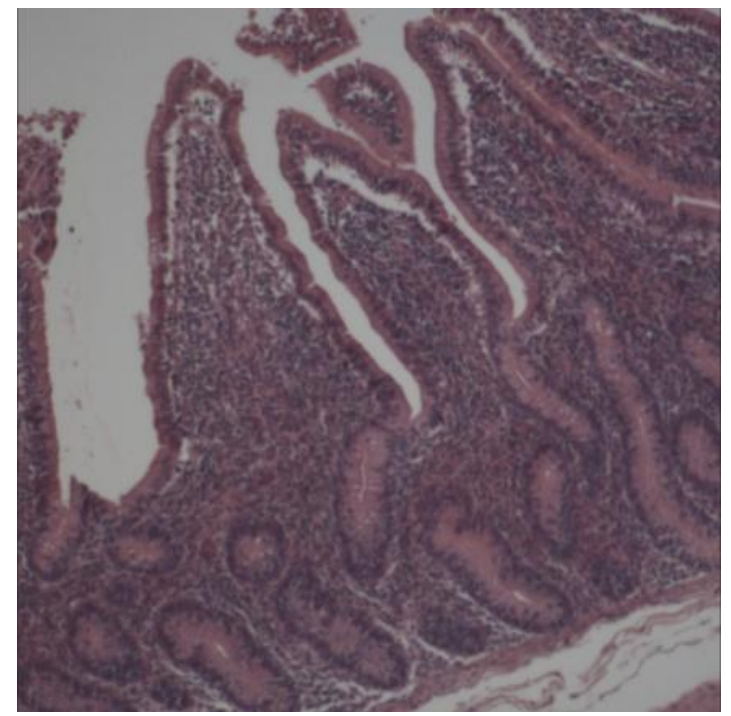

Figura 2. Vilosidades e criptas do intestino delgado (10x; HE) de cordeiros Santa Inês, submetidos ao regime alimentar para ganho compensatório, terminados em confinamento.

provavelmente na tentativa de aumentar a superfície de absorção, já que o intestino delgado parece se adaptar para satisfazer as necessidades 
nutricionais do animal. Essa manobra atenderia a necessidade de aumentar a procura de nutrientes (Zitnan et al., 2008) e de compensar a baixa ingestão desses elementos pelos cordeiros durante o período de restrição prévia, pois uma menor área da vilosidade implicaria menor atividade enzimática, redução na digestibilidade e absorção de nutrientes (Arruda et al., 2008).
Elevadas correlações, altamente significativas $(\mathrm{P}<0,01)$, foram encontradas entre as variáveis ganho de peso, consumo, peso ao abate, peso do corpo vazio e peso do trato gastrintestinal (Tab. 5). Correlações menores, no entanto significativas $(\mathrm{P}<0,05)$, foram observadas entre o peso dos componentes abióticos e essas variáveis.

Tabela 5. Correlação entre ganho de peso (GPMD, GPT), consumo (CMS, CPB, CEB), peso ao abate (PA), peso do corpo vazio (PCV), peso dos componentes abióticos (PCA) e trato gastrintestinal (TGI) de cordeiros Santa Inês, submetidos ao regime alimentar para ganho compensatório, terminados em confinamento

\begin{tabular}{lcccccccc}
\hline Var. & GPT & CMS & CPB & CEB & PA & PCV & PCA & TGI \\
\hline GPMD & $1,00^{* *}$ & $0,86^{* *}$ & $0,86^{* *}$ & $0,86^{* *}$ & $0,95^{* *}$ & $0,90^{* *}$ & $0,62^{* * *}$ & $0,73^{* *}$ \\
GPT & - & $0,86^{* *}$ & $0,86^{* *}$ & $0,86^{* *}$ & $0,95^{* *}$ & $0,91^{* *}$ & $0,62^{* *}$ & $0,73^{* *}$ \\
CMS & & - & $1,00^{* *}$ & $1,00^{* * *}$ & $0,89^{* *}$ & $0,91^{* *}$ & $0,33^{*}$ & $0,74^{* *}$ \\
CPB & & & - & $1,00^{* *}$ & $0,89^{* *}$ & $0,92^{* *}$ & $0,33^{*}$ & $0,75^{* *}$ \\
CEB & & & & - & $0,89^{* *}$ & $0,92^{* *}$ & $0,34^{*}$ & $0,74^{* *}$ \\
PA & & & & & - & $0,97^{* *}$ & $0,56^{* *}$ & $0,81^{* *}$ \\
PCV & & & & & & - & $0,39^{*}$ & $0,84^{* *}$ \\
PCA & & & & & & & - & $0,33^{*}$ \\
\hline
\end{tabular}

Var. $=$ variáveis. $\mathrm{P}<0,01^{* *} ; \mathrm{P}<0,05^{*}$.

Correlações positivas entre as variáveis de desempenho produtivo estudadas também foram encontradas por Barroso et al. (2006), em ovinos SRD alimentados com dietas contendo resíduo desidratado de vitivinícolas. O maior consumo de MS e PB resultou em melhor desempenho dos animais, o que confirma que as taxas de ganho de peso de ovinos são dependentes dos níveis de consumo (Kozloski et al., 2006).

A correlação positiva encontrada entre os ganhos de peso (médio diário e total), os consumos, o peso ao abate e os pesos do corpo vazio e do TGI também foi observada por Shadnoush et al. (2011), em ovinos submetidos à restrição alimentar. Esses autores afirmam haver uma correlação entre o consumo e o peso dos órgãos, o que é complementado por Yamamoto et al. (2004), quando sugerem que os órgãos se desenvolvem similarmente com o aumento do peso vivo do animal.

O peso do TGI e os consumos de matéria seca, proteína e energia tiveram índices de correlação positiva e significativa $(\mathrm{P}<0,05$ e 0,01$)$, apenas com largura e área das papilas ruminais (Tab. 6). Álvarez-Rodríguez et al. (2012) afirmam que a morfologia do rúmen se adapta ao tipo de alimento. Entretanto considerando que, no presente experimento, a alimentação permaneceu a mesma durante todo o período experimental, variando apenas a quantidade oferecida na fase de restrição prévia, sugere-se que a subalimentação provocou uma redução no tamanho das papilas ruminais e que a retomada do seu desenvolvimento ocorreu lentamente durante a fase de realimentação, conforme afirmam Martens et al. (2012).

Entre as medidas morfométricas do rúmen, houve correlação positiva e altamente significativa $(\mathrm{P}<0,01)$ da área das papilas ruminais com altura e largura. Da mesma forma ocorreu no intestino delgado, onde foi observada correlação positiva $(\mathrm{P}<0,01)$ entre a área das vilosidades com altura, largura e superfície, assim como da altura das vilosidades com a superfície. Correlação positiva entre as medidas das papilas também foi observada por Sun et al. (2011), em bovinos suplementados com probiótico, e entre as medidas de vilosidades, por Blättler et al. (2001), ao avaliarem o consumo de colostro com sucedâneo em bezerros, o que confirma a alta relação entre essas medidas, que, quando aumentam, resultam em maior superfície absortiva, e o consequente aumento na capacidade de absorção de nutrientes. 
Regime alimentar para ganho...

Tabela 6. Correlação entre peso do trato gastrintestinal, consumo e medidas morfométricas do rúmen e do intestino de cordeiros Santa Inês, submetidos ao regime alimentar para ganho compensatório, terminados em confinamento

\begin{tabular}{lcccccccc} 
Var. & APR & LPR & ARPR & AVI & LVI & SVI & ARVI & PCI \\
\hline TGI & 0,21 & $0,39^{* *}$ & $0,36^{*}$ & 0,08 & $-0,18$ & 0,08 & $-0,05$ & $-0,16$ \\
CMS & 0,07 & $0,54^{* *}$ & $0,36^{*}$ & $-0,07$ & $-0,12$ & $-0,07$ & $-0,18$ & $-0,23$ \\
CPB & 0,09 & $0,55^{* *}$ & $0,38^{*}$ & $-0,07$ & $-0,11$ & $-0,07$ & $-0,17$ & $-0,23$ \\
CEB & 0,07 & $0,54^{* *}$ & $0,36^{*}$ & $-0,07$ & $-0,12$ & $-0,07$ & $-0,18$ & $-0,23$ \\
APR & - & 0,20 & $0,77^{* *}$ & $-0,18$ & 0,21 & $-0,17$ & $-0,02$ & 0,29 \\
LPR & & - & $0,63^{* *}$ & $-0,19$ & 0,14 & $-0,20$ & $-0,16$ & $-0,03$ \\
ARPR & & & - & $-0,21$ & 0,26 & $-0,20$ & $-0,07$ & 0,12 \\
AVI & & & & - & $-0,16$ & $0,99^{* *}$ & $0,77^{* *}$ & 0,24 \\
LVI & & & & - & $-0,04$ & $0,47^{* *}$ & $-0,02$ \\
SVI & & & & & - & - & $0,84^{* *}$ & 0,22 \\
ARVI & & & & & & & - & 0,17 \\
\hline Var.
\end{tabular}

Var. = variáveis; TGI = peso do trato gastrintestinal CMS, CPB e CEB = consumo de matéria seca, proteína bruta e energia bruta; APR, LPR e ARPR = altura, largura e área das papilas ruminais; AVI, LVI, SVI e ARVI = altura, largura, superfície e área das vilosidades do intestino; PCI = profundidade das criptas do intestino. $\mathrm{P}<0,01^{* * *}$; $\mathrm{P}<0,05^{*}$.

\section{CONCLUSÕES}

O regime alimentar para ganho compensatório diminui o ganho de peso, o peso ao abate, o peso do corpo vazio e o peso do trato gastrintestinal de cordeiros Santa Inês, terminados em confinamento, além de diminuir a largura das papilas ruminais e aumentar a área das vilosidades do intestino delgado. Elevadas correlações positivas foram observadas entre ganho de peso, consumo, peso ao abate, peso do corpo vazio e peso do trato gastrintestinal, da mesma forma de peso do trato gastrintestinal e consumo, com largura e área das papilas ruminais.

\section{REFERÊNCIAS}

ALMEIDA, T.R.V.; PEREZ, J.R.O.; CHLAD, M. et al. Desempenho e tamanho de vísceras de cordeiros Santa Inês após ganho compensatório. Rev. Bras. Zootec., v.40, p.616-621, 2011.

ÁLVAREZ-RODRÍGUEZ， J.; MONLEÓN， E.; SANZ, A. et al. Rumen fermentation and histology in light lambs as affected by forage supply and lactation length. Res. Vet. Sci., v.92, p.247-253, 2012.

ARRUDA, A.M.V.; FERNANDES, R.T.V.; SILVA, J.M.; LOPES, D.C. Avaliação morfo-histológica da mucosa intestinal de coelhos alimentados com diferentes níveis e fontes de fibra. Caatinga, v.21, p.01-11, 2008.
BARROSO, D.D.; ARAÚJO, G.G.L.; SILVA, D.S. et al. Desempenho de ovinos terminados em confinamento com resíduo desidratado de vitivinícolas associado a diferentes fontes energéticas. Cienc. Rural, v.36, p.1553-1557, 2006.

BEN SALEM, H.; SMITH, T. Feeding strategies to increase small ruminant production in dry environments. Small Rumin. Res., v.77, 174-194, 2008.

BLÄTTLER, U.; HAMMON, H.M.; MOREL, C. et al. Feeding colostrum, its composition and feeding duration variably modify proliferation and morphology of the intestine and digestive enzyme activities of neonatal calves. J. Nutr., v.131, p.12561263, 2001.

BORBUREMA, J.B. Desempenho e perfil metabólico de ovinos Santa Inês submetidos ao regime alimentar para crescimento compensatório em confinamento. 2010. 56f. Dissertação (Mestrado em Medicina Veterinária) - Centro de Saúde e Tecnologia Rural, Universidade Federal de Campina Grande, Patos, PB.

HOMEM JUNIOR, A.C.; EZEQUIEL, J.M.B.; GALATI, R.L. et al. Grãos de girassol ou gordura protegida em dietas com alto concentrado e ganho compensatório de cordeiros em confinamento. Rev. Bras. Zootec., v.39, p.563-571, 2010.

HOMEM JUNIOR, A.C.; SILVA SOBRINHO, A.G.; YAMAMOTO, S.M. et al. Ganho compensatório em cordeiras na fase de recria: desempenho e medidas biométricas. Rev. Bras. Zootec., v.36, p.111-119, 2007.

INSTITUTO BRASILEIRO DE GEOGRAFIA E ESTATÍSTICA. Produção da Pecuária Municipal. Rio de Janeiro, v.39, 2011. 63p. 
JENKINS, T.C. Lipid metabolism in the rumen. $J$. Dairy Sci., v.76, p.3851-3863, 1993.

KAMALZADEH, A.; KOOPS, W.J.; VAN BRUCHEM, J. et al. Feed quality restriction and compensatory growth in growing sheep: development of body organs. Small Rumin. Res., v.29, p.71-82, 1998.

KOZLOSKI, G.V.; TREVISAN, L.M.; BONNECARRÈRE, L.M. et al. Níveis de fibra em detergente neutro na dieta de cordeiros: consumo, digestibilidade e fermentação ruminal. Arq. Bras. Med. Vet. Zootec., v.58, p.893-900, 2006.

MARTENS, H.; RABBANI, I.; SHEN, Z. et al. Changes in rumen absorption processes during transition. Anim. Feed Sci. Technol., v.172, p.95-102, 2012.

MEDEIROS, G.R.; CARVALHO, F.F.R.; FERREIRA, M.A. et al. Efeito dos níveis de concentrado sobre os componentes não-carcaça de ovinos Morada Nova em confinamento. Rev. Bras. Zootec., v.37, p.1063-1071, 2008

MORENO, G.M.B.; SILVA SOBRINHO, A.G.; LEÃO, A.G. et al. Rendimento dos componentes nãocarcaça de cordeiros alimentados com silagem de milho ou cana-de-açúcar e dois níveis de concentrado. Rev. Bras. Zootec., v.40, p.2878-2885, 2011.

NATIONAL RESEARCH COUNCIL - NRC. Nutrient requeriments of sheep. 6.ed. Washington, D.C.: National Academy Press, 1985. 99p.

NATIONAL RESEARCH COUNCIL - NRC. Nutrient requirements of small ruminants: sheep, goats, cervids, and new world camelids. Washington, D.C.: National Academies Press, 2007. 347p.

NOROUZIAN, M.A.; VALIZADEH, R.; VAHMANI, P. Rumen development and growth of Balouchi lambs offered alfalfa hay pre- and post-weaning. Trop. Anim. Health Prod., v.43, p.1169-1174, 2011.

OWENS, F.N.; DUBESKI, P.; HANSON, C.F. Factors that alter the growth and development of ruminants. J. Anim. Sci., v.71, p.3138-3150, 1993.
RYAN, W.J. Compensatory growth in cattle and sheep. Nutr. Abstr. Rev. (Series B), v.60, p.653-664, 1990.

SAMUELSON, D.A. Tratado de Histologia Veterinária. RJ: Elsevier, 2007. 527p.

SHADNOUSH, G.R.; ALIKHANI, M.; RAHMANI, H.R. et al. Effects of restricted feeding and re-feeding in growing lambs: intake, growth and body organs development. J. Anim. Vet. Adv., v.10, p.280-285, 2011.

SILVA, D.J.; QUEIROZ, A.C. Análise de alimentos: métodos químicos e biológicos. 3.ed. Viçosa: Editora UFV, 2002. 235p.

STATISTICAL ANALISYS SYSTEM INSTITUTE. User's guide. North Caroline: SAS Institute Inc., 2003.

SUN, P.; WANG, J.Q.; ZHANG, H. Effects of supplementation of Bacillus subtilis natto $\mathrm{Na}$ and $\mathrm{N} 1$ strains on rumen development in dairy calves. Anim. Feed Sci. Technol., v.164, p.154-160, 2011.

WANG, Y.H.; XU, M.; WANG, F.N. et al. Effect of dietary starch on rumen and small intestine morphology and digesta $\mathrm{pH}$ in goats. Liv. Sci., v.122, p.48-52, 2009.

XU, M.; DONG, Y.; DU, S. et al. Effect of corn particle size on mucosal morphology and digesta $\mathrm{pH}$ of the gastrointestinal tract in growing goats. Liv. Sci., v.123, p.34-37, 2009.

YAMAMOTO, S.M.; MACEDO, F.A.F.; MEXIA, A.A. et al. Rendimentos dos cortes e não-componentes das carcaças de cordeiros terminados com dietas contendo diferentes fontes de óleo vegetal. Cienc. Rural, v.34, p.1909-1913, 2004.

ZITNAN, R.; VOIGT, J.; KUHLA, S. et al. Morphology of small intestinal mucosa and intestinal weight change with metabolic type of cattle. Vet. Med., v.53, p.525-532, 2008. 